\title{
COMPOSIÇÃO DA MIMERCOFAUNA DE UM FRAGMENTO DE CERRADO EM REGENERAÇÃO EM BOTUCATU, SÃO PAULO
}

\author{
Macrofauna composition of a cerrado fragment in regeneration in \\ Botucatu, São Paulo \\ Composición de la macrofauna de un fragmento cerrado en la regeneración de \\ Botucatu, São Paulo
}

\author{
Maria Ines Bulgari Alves \\ Mestranda, UNESP, Brasil \\ bulgari@ibb.unesp.br
}

José Luiz Chiaradia Gabriel Professor Doutor, FREA, Brasil. zulubot@yahoo.com.br 


\section{RESUMO}

O Cerrado é o segundo maior bioma da América do sul e o mais abundante no Brasil, possui uma grande diversidade de nichos, fauna e flora. Esse bioma vem desaparecendo e estudo em áreas ambientais estão sendo desenvolvidos para acompanhar o processo de regeneração. O objetivo desse trabalho é buscar conhecer a composição de formigas em um fragmento de cerrado que está se regenerando no município de Botucatu, SP, que se localiza em área de preservação ambiental (EMA). Foram realizadas nove coletas uma por mês em cinco pontos do fragmento, no período de março a novembro de 2013, totalizando 45 amostras. Para coletar a fauna de formigas utilizamos o amostrador tipo Pittfall, que ficavam expostos por 24 horas e após eram retirados, e o material coletado era armazenado em álcool $70 \%$ e após transferido para o laboratório onde as formigas foram triadas e identificadas sobre esteriomicroscópio. Coletamos 4.532 indivíduos, sendo sua frequência amostral maior no mês de junho com 634 indivíduos e a menor em maio com 357 indivíduos. A riqueza observada foi de 19 espécies sendo distribuídas em 12 gêneros e 4 subfamílias. Os gêneros Camponitinni e Ectatommini destacaram se pelo registro de 4 espécies cada, ainda esses gêneros foram os mais abundantes em número de indivíduos coletados em todas as amostras. Pelo registro de espécies dos gêneros Camponitinni e Brachymyrmex que são sensíveis a mudanças no ambiente podendo se constatar que o fragmento de cerrado está se regenerando, esses gêneros são bioindicadoras de qualidade ambiental de diversos biomas.

PALAVRAS-CHAVE: biomas, diversidade, abundância, preservação.

\section{ABSTRACT}

The Cerrado is the second largest biome in South America and the most abundant in Brazil, has a wide variety of niches, fauna and flora. This biome is disappearing and study in environmental areas are being developed to monitor the regeneration process. The aim of this work is to seek to know the composition of ants in a fragment of savannah that is regenerating in Botucatu, SP, located in environmentally protected area (EMA). nine samples were taken once a month at five points of the fragment, in the period from March to November 2013, totaling 45 samples. To collect the ant fauna use the sampler Pittfall, which were exposed for 24 hours after they were removed, and the collected material was stored in $70 \%$ alcohol and after transferred to the laboratory where the ants were screened and identified on esteriomicroscópio. We collected 4,532 individuals, a larger sample frequency in June with 634 individuals and the lowest in May with 357 individuals. The observed richness was 19 species being distributed in 12 genera and 4 subfamilies. The Camponitinni and Ectatommini genres highlighted is the record of 4 species each, although these genres were the most abundant in number of individuals in all samples. The registration of species of Camponitinni and Brachymyrmex genres that are sensitive to changes in the environment can be seen that the cerrado fragment is regenerating, these genres are bioindicators of environmental quality of various biomes

KEYWORDS: biomes, diversity, abundance, preservation.

\section{RESUMEN}

El Cerrado es el segundo mayor bioma en América del Sur y el más abundante en Brasil, tiene una amplia variedad de nichos, fauna y flora. Este bioma está desapareciendo y el estudio en áreas ambientales se están desarrollando para controlar el proceso de regeneración. El objetivo de este trabajo es tratar de conocer la composición de hormigas en un fragmento de la sabana que se regenera en Botucatu, SP, situado en zona de protección medioambiental (EMA). nueve muestras se tomaron una vez al mes a cinco puntos del fragmento, en el período de marzo a noviembre de 2013, por un total de 45 muestras. Para recoger la fauna de hormigas utilizan el sampler Pittfall, que fueron expuestos durante 24 horas después de que fueron removidos, y el material obtenido se almacenó en alcohol al $70 \%$ y después trasladado al laboratorio donde fueron seleccionados e identificados en esteriomicroscópio las hormigas. Hemos recogido 4.532 personas, una frecuencia mayor de muestras en junio con 634 personas y el más bajo en mayo con 357 individuos. La riqueza observada fue de 19 especies que se distribuyen en 12 géneros y 4 subfamilias. Los géneros Camponitinni y Ectatommini resaltan es el registro de 4 especies cada uno, a pesar de estos géneros fueron los más abundantes en número de individuos en todas las muestras. El registro de especies de Camponitinni y Brachymyrmex géneros que son sensibles a los cambios en el medio ambiente puede ser visto que el fragmento de cerrado está regenerando, estos géneros son bioindicadores de calidad ambiental de varios biomas.

PALABRAS-CLAVE: biomas, diversidad, abundancia, la preservación. 


\section{INTRODUÇÃO}

O Cerrado é o segundo maior bioma da América do sul e o mais abundante no Brasil, abrangendo cerca de $22 \%$ do território brasileiro. É um conjunto de ecossistemas que abrange savanas, matas, campos e matas de galeria (EINTEN 1977, EINTEN 1987; RIBEIRO et al., 1998; MACHADO et.al 2004).

Esse bioma tem sido extinto pela prática da agricultura, sendo a soja o produto mais produzido nessas áreas, e para que essas culturas se estabeleçam os produtores desmatam e enriquecem o solo com fertilizantes e calcários (MARCHÃO, 2009) Além da agricultura a produção de pastos para a bovinocultura também é um dos principais agravantes da devastação desse ambiente (PRIMACK \& RODRIGUES, 2002).

A degradação está ocorrendo em tempo recorde, cerca de $60 \%$ desse bioma já foi extinto, e estima-se que o tamanho da sua área desmatada equivale à área da floresta Amazônica. (KLINK \& MOREIRA, 2002). No Estado de São Paulo esse bioma é o mais antropotizado, e essa situação vem ocorrendo em praticamente todo o seu território (KRONKA et al. 2005). A área conservada restante é de $13 \%$ de cobertura vegetal natural desse bioma (MACHADO et al., 2004).

O desmatamento e a redução do mesmo vêm afetando ecossistema e extinguindo espécies endêmicas (REDFORD et al, 1990; MACHADO et al., 2004). A diversidade tanto animal como vegetal estão sofrendo com essa degradação diariamente, esse bioma é uma área importante pois possui uma variação ambiental marcante e uma grande diversidade de nichos, fauna e flora (COUTINHO, 1976; KLINK \& MACHADO, 2005).

Por essa variação fornece diferentes habitats para as formigas, que são insetos sociais e com uma grande diversidade de gêneros, que se adaptam a todos os ambientes, não estando presente apenas nos polos, é o inseto com a maior derivação de gêneros depois dos coleópteros (BORROR \& DELONG, 1969; FORTI \& BOARETTO, 1997; SANTOS et al, 2003).

As colônias de formigas podem ser simples com um número mínimo de indivíduos e também podem chegar a milhares delas em uma única colônia (WILSON,1971). Elas possuem diversos hábitos alimentares, podendo ser carnívoras, se alimentarem de secreções liquidas, sementes, fungos, animais vivos e mortos (FOWLER et al, 1991).

Diversos autores tem demostrado que entre uma variedade de insetos as formigas podem ser utilizadas como bioindicadoras de qualidade do ambiente (MARINHO et al. 2002; ARAÚJO et al. 2004; BICKEL \& WATANASIT 2005), são sensíveis a mudanças de clima perda de vegetações ou até mesmo de espécies da qual elas se alimentam (BUZZI \& MIYAZAKI,1999).

Muitas pesquisas estão ligadas as formigas, porém poucas veem esses insetos como bioindicadores da regeneração de ambientes. O objetivo desse trabalho é buscar conhecer a composição da fauna de formigas em um fragmento de cerrado que está se regenerando no município de Botucatu, SP, em uma área de preservação ambiental de Botucatu (EMA). 


\section{MATERIAIS E MÉTODOS}

\section{1. Área de estudo}

O Fragmento de cerrado está localizado na cidade de Botucatu, SP (48²7'22.44"O e $22^{\circ} 55^{\prime} 36.33^{\prime \prime S}$ ) em uma área de proteção ambiental onde se encontra as instalações da Escola do Meio Ambiente (EMA) instituição pertencente à Prefeitura municipal de Botucatu-SP, criada em 2005, através da Secretaria Municipal de Educação, para formação de educadores ambientais. A área de cerrado da escola ocorre em formas de manchas, sendo cercado com remanescentes de florestas estacional semidecídual, com arbustos pequenos e algumas árvores maiores, seu solo possui uma cobertura de $70 \%$ de grama braquiária (Figura 1).

Figura 1(A-B) Vista aérea do fragmento de Cerrado de Botucatu/SP

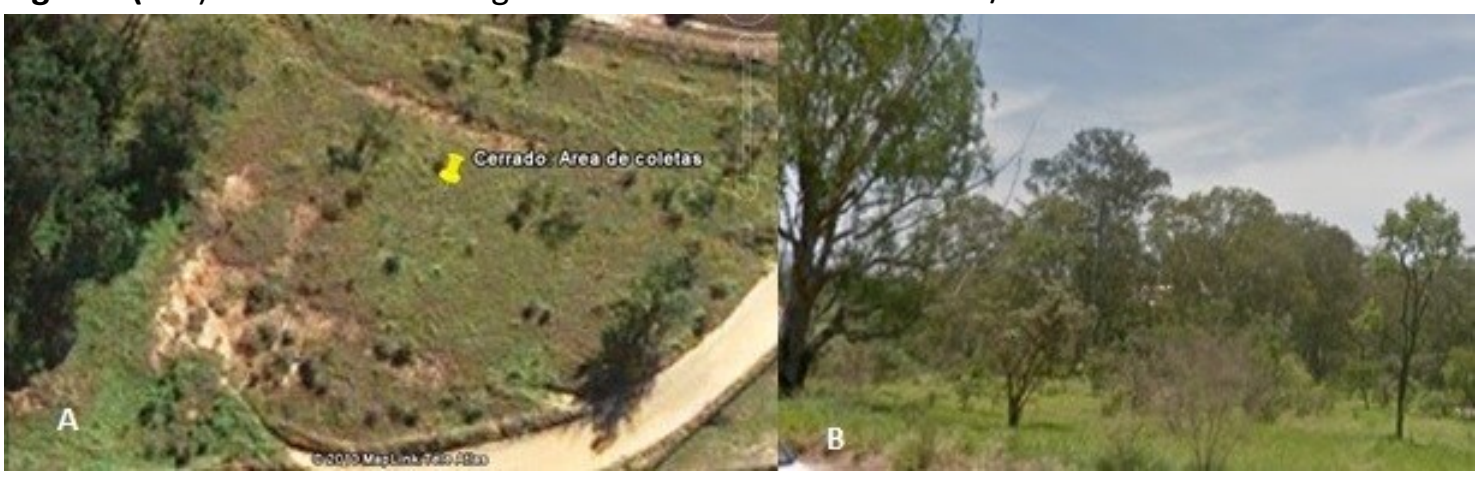

FONTE: GOOGLE EARTH(2013)

\subsection{Metodologia}

As coletas foram realizadas de março a novembro 2013, com armadilhas de Pittfall que são compostas de potes de plástico transparente de $500 \mathrm{ml}$, nesses potes eram colocados $200 \mathrm{ml}$ de água com sabão liquido neutro, para que as formigas assim que capturadas não conseguissem sair da armadilha. Para a instalação dos Pittfall foi seguido à metodologia de PARR \& CHOWN, 2001. As armadilhas ficaram expostas durante 24 horas, em cinco pontos do cerrado distribuídos aleatoriamente, eram realizadas uma vez por mês. Após esse material era triado e identificado com auxílio de esteriomicroscópio, Para a identificação usamos chaves de apropriadas e colaboração de profissionais de entomologia do laboratório de Insetos Sociais do Departamento de Proteção de Plantas, FCA-UNESP, Botucatu/SP.

\section{RESULTADOS}

Foram identificados e quantificados 4.532 indivíduos sendo distribuídos em 12 gêneros, pertencentes a quatro subfamílias e a 19 espécies. A subfamília Ponerinae foi a mais abundante em número de espécies registradas no fragmento de cerrado sendo quatro pertencentes ao 
gênero Ectatommini, e quatro pertencente ao gênero Camponotini e registrou também o gênero Ponerini, com duas espécies (Tabela 1).

Tabela 1. Subfamílias, gêneros e espécies amostradas no fragmento de Cerrado em Botucatu, SP.

SUBFAMÍLIAS

SUBFAMÍLIA PONERINAE

SUBFAMÍLIA FORMICINAE

SUBFAMILIA MYRMICINAE

SUBFAMÍLIA

PSEUDOMYRMICINAE

Fonte: DO AUTOR, 2016

\section{GÊNEROS E ESPÉCIES}

\section{ECTATOMMINI}

Ectatomma brunneum Fr. (SMITH)

Ectatomma edentatum (ROGER)

Ectatomma permagnum (FOREL)

Ectatomma tuberculatum (OLIVER)

CAMPONOTINI

Camponotus crassus (MAYR)

Camponotus fastigatus (ROGER)

Camponotus latangulus (ROGER)

Camponotus melanoticus (EMERY)

PONERINI

Odontomachus bauri (EMERY)

Pachycondyla apicalis (LATREILLE)

BRACHYMYRMECINI

Brachymyrmex depilis

ATTINI - ACROMYRMEX

Acromyrmex balzani (EMERY)

ATTINI-ATTA

Atta sexdens rubropilosa (FOREL)

Atta sexdens sexdens (LINNAEUS)

BLEPHARIDATTINI

Blepharidatta (KEMP, 1967)

CREMATOGASTRINI

Crematogaster spp

PHEIDOLINI

Pheidole fallax (MAYR)

SOLENOPSIDINI

Solenopsis saevissima (FR. SMITH)

PSEUDOMYRMECINI

Pseudomyrmex gracilis (FABRICIUs) 
Os gêneros da subfamília Ponerinae foram o mais abundantes em número de espécies registraram juntos 10 espécies, a subfamília Myrmicinae foi o mais abundante em número de gêneros registrando 6 gêneros e 7 espécies(tabela 1). A Subfamília Pseudomyrmicinae e a subfamília Formicinae, registrarão um gênero e uma espécie para ambas as subfamílias.

O gênero Ectatomini registrou 1347, sendo o mais abundante seguido por Camponotini com 974 e Solenopsidini com 515 indivíduos. (tabela 2)

Tabela 2. Abundância de indivíduos por gêneros de formigas coletados no fragmento de Cerrado de Botucatu/ SP

\begin{tabular}{lc}
\hline Genêros de formigas & Número de indivíduos \\
\hline Ectatommini & 1347 \\
Camponotini & 974 \\
Ponerini & 610 \\
Brachymyrmecini & 205 \\
Acromyrmex & 106 \\
Atta & 109 \\
Blepharidattini & 143 \\
Crematogastrini & 235 \\
Pheidolini & 167 \\
Solenopsidini & 515 \\
Pseudomyrmecini & 124 \\
\hline
\end{tabular}

Fonte: DO AUTOR, 2016

Formigas do gênero Brachymyrmecini, tiveram registradas apenas uma espécie, com um número de 205 indivíduos, ainda também com apenas uma espécie o gênero Pheidolini, esse gênero é considerado bioindicador junto com as Solenopsinnis de ambientes degradados. Esses gêneros foram amostrados em número de indivíduos relevante.

Analisamos a abundância de indivíduos coletadas por amostra e comparamos entre os meses, o mês de junho apresentou um número de individuo elevado de 634 indivíduos e o mês de maio foi o que menos ocorreu indivíduos capturados com 357(figura 2). 
Figura 2. Abundância de indivíduos coletados mensalmente

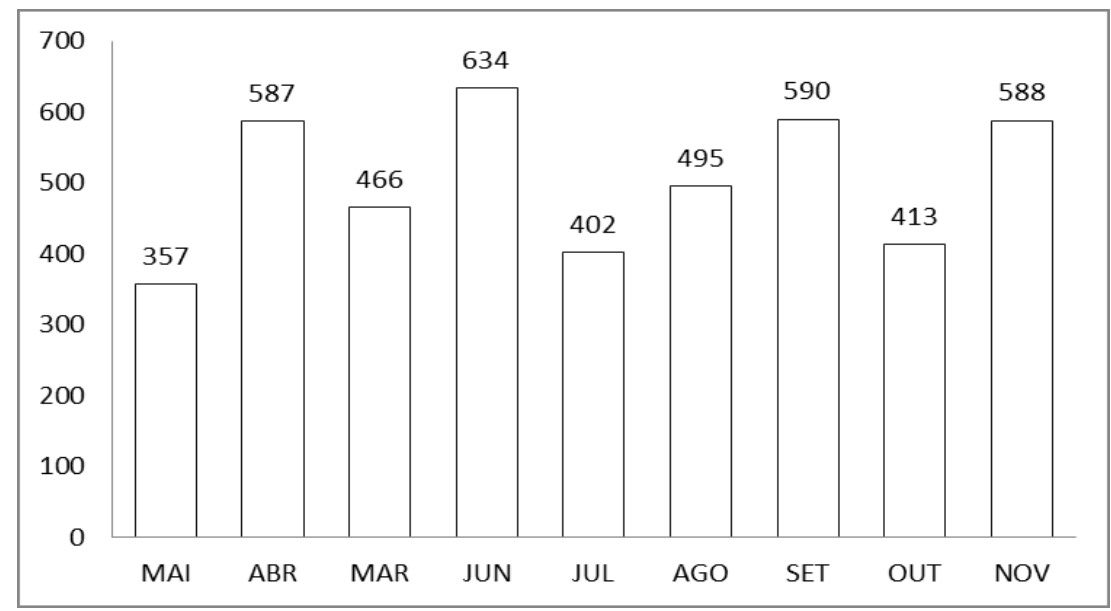

Fonte: DO AUTOR, 2016

Mensuramos também a riqueza observada, riqueza estimada e a variância, dos gêneros em relação aos meses amostrados através do teste de riqueza de Jackkinife de $1^{\circ}$ ordem, mensurada através do softwer DIVES (figura3). A riqueza estimada variou de 11 no mês de março a 20 no mês de novembro, ainda a riqueza observada não se modificou, pois todos os gêneros foram amostrados em todos os meses de coletas, a variância foi de $(0,75$ á 1,68$)$

Figura 3. Riqueza de Jackkinife de $1^{\circ}$ ordem, mensurada para riqueza observada, riqueza estimada e variância.

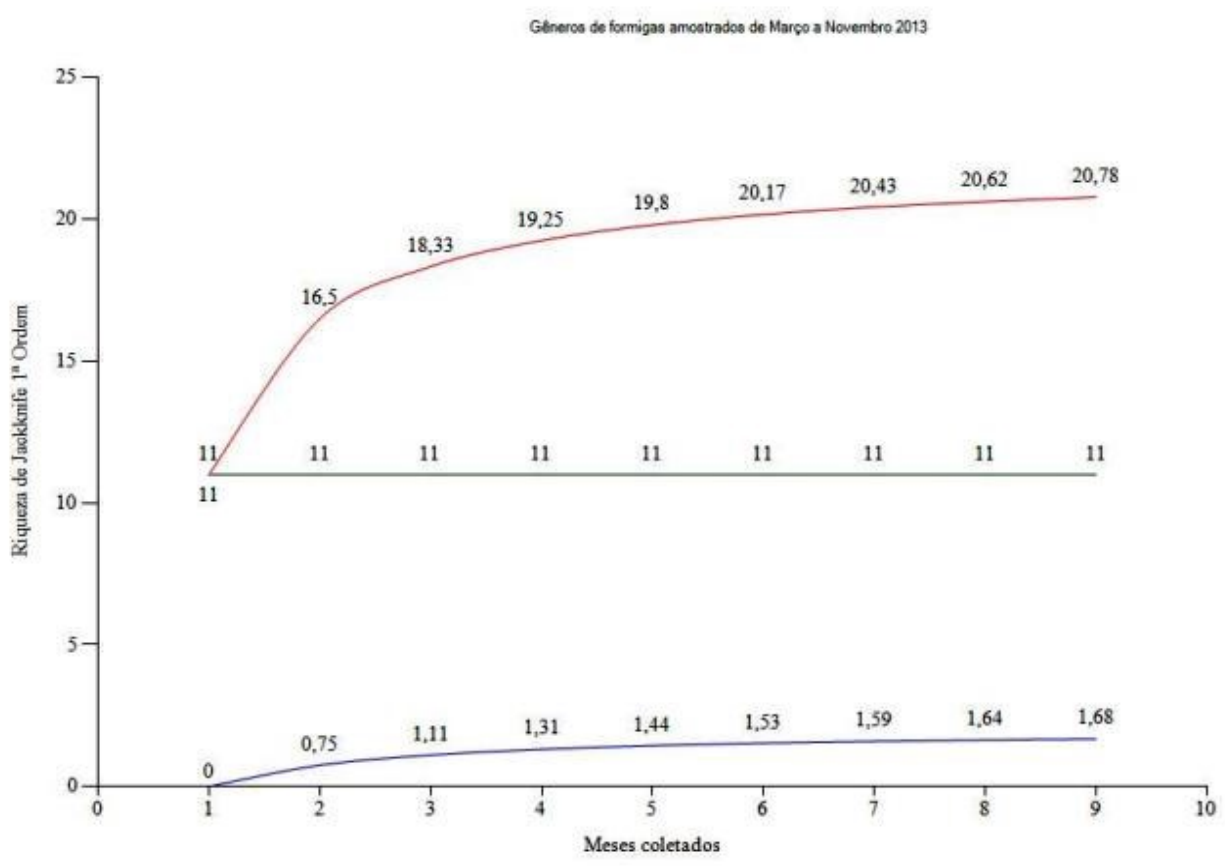

FONTE: DO AUTOR, 2016 


\section{DISCUSSÃO}

O fragmento de cerrado estudado estava degradado, pois utilizavam esse local para deposito ilegal de lixo após ser integrado à área de proteção ambiental foi retirados os entulhos, e até então ele vem regenerando sua flora e fauna vagarosamente.

A composição da fauna de formigas desse ambiente quando comparadas com trabalhos realizados em outras áreas de Cerrados consideradas conservadas podemos observar alguns gêneros como Camponotinii, Ectatommini, Pheidolini, que foram registrados em nosso trabalho e também foram registrados em um levantamento no Parque Estadual da Serra de Caldas novas (SILVESTRE, 2000).

CAMPOS et al(2004) em um levantamento comparativo de áreas registrou para o campo de cerrado os gêneros Camponotinni e Ectatommini, que colaboram com o nosso levantamento no fragmento pois esses gêneros são muito abundantes em diversidade de espécies, extremamente generalistas na procura de alimento

As formigas das espécies Camponotus spp e Pseudomyrmex, spp, forrageiam, em todas as partes atrás de alimentos, essas podem chegar a patrulhar uma área muito grande longe do seu ninho de origem, amostramos essas espécies e constatamos o grande número de indivíduos que podem ter ocorrido por esse motivo de busca de alimento (MARINHO et.al 2002). Por estar presente nesse ambiente com o número de espécies e abundante em número de indivíduos o gênero Camponotini indica que o fragmento de cerrado está se reestabelecendo, pois esse gênero possui espécies que são dependentes parcialmente de pulgões que são sensíveis a degradação.

Formigas são animais com diversos hábitos de forrageamento, possuem diversas estratégias alimentares, por esse motivo conseguimos capturar gêneros diversos como Atta e Acromyrmex que são gêneros que cultivam fungo simbionte, através de folhas para posteriormente se alimentarem deles (FORTI \& BOARETTO, 1997). Os indivíduos desses gêneros são forrageadoras ativas, saem para buscar novas fontes de alimentos e por esse motivo devem ter caído nas armadilhas. Os demais gêneros encontrados são forrageadores vorazes, buscam todos os tipos de alimentos para a colônia salvando algumas espécies de Camponotini, que são associadas a pulgões e se alimentam das secreções que esses produzem em troca de que elas os protejam de predadores (BORROR \& DELONG, 1969, MARINHO; C. 2002).O gênero de Solenopsinni, foram amostrados com uma frequência considerada mínima, mas a ocorrência desse genro pode indicar a degradação desse fragmento pois essas conseguem estabelecer sua colônia mesmo com pouco recurso disponível, elas competem com outras espécies por alimento, pois podem recrutar um número grande de indivíduos para coletar alimento (DELABIE \& FOWLER 1995; FOWLER et al. 1991).

Estudos com biomonitoramento verificaram que as formigas são boas indicadoras da degradação de diversos ecossistemas, variando o número de indivíduos conforme as condições ambientais, sendo que colônias com um número elevado de indivíduos reduzem ao mínimo para que consigam sobreviver nesses ambientes degradados (MAJER 1993).No nosso trabalho 
encontramos uma abundancia de gêneros considerada pequena para áreas de Cerrado pois já se registram cerca de 52 gêneros, em áreas conservadas, o baixo número pode indicar que o fragmento de cerrado está se regenerando, e as comunidades de formigas estão reestabelecendo. Nesse fragmento de cerrado foram encontrados poucos gêneros, porém esse levantamento de fauna indica a regeneração desse bioma, e assim podemos entender como ele ocorre através das formigas, conforme aumenta o número de gêneros encontrados, indica que esse bioma está se regenerando, pois áreas degradadas podem possuir um número menor de gêneros de formigas. As espécies que utilizam de espécies de pulgões estão fortemente associadas a ambientes preservados, como as Camponotini, quando não encontradas nesses ambientes podem ser um sinal de degradação elevada (FERNÁNDEZ, F, 2003) os gêneros de Pheidole, Solenopsis e Acromyrmex são comuns, sendo facilmente encontradas em sistemas de produção degradados.

\section{CONCLUSÃO}

O presente trabalho colabora com dados iniciais de regeneração desse fragmento de cerrado de Botucatu, sendo que este está vagarosamente se reestabelecendo.

Formigas são boas indicadoras tanto de regeneração por seus gêneros sensíveis a degradação e também pelos gêneros que se estabelecem em áreas mesmo que degradadas.

O fragmento de cerrado estudado ainda precisa de mais acompanhamentos em relação a essa fauna para que se possa determinar se ele se restabeleceu, e é importante salientar que esse trabalho contribuiu com informações pra conservação desse fragmento.

\section{REFERÊNCIAS BIBLIOGRÁFICAS}

BORLAUG, N.E. Feeding a world of 10 billion people: the miracle ahead. In: R. Bailey (ed.). Global warming and other eco-myths. p. 29-60. Competitive Enterprise Institute, Roseville, EUA, 2002.

BORROR, D. J. \& DELONG, D.M. Introdução ao estudo dos insetos São Paulo: Edgard Blucher, p.656, 1969.

BUZZI, Z. J \& MIYAZAKII, R. D. Entomologia didática. 3o ed., Ed. UFPR, Curitiba PR, 308 p. 1999

CAVASSAN, O. O cerrado do Estado de São Paulo. In: KLEIN, A. L. (Org.). Eugen Warming e o cerrado brasileiro: um século depois. São Paulo: UNESP. p.93-106, 2002

COUTINHO, L.M. Contribuição ao conhecimento do papel ecológico das queimadas na floração de espécies do cerrado. Tese (Livre Docência). Instituto de Biociências , Universidade de São Paulo, São Paulo, 1976.

DELABIE, J.H.C \& H.G. FOWLER. Soil and litter cryptic ant assemblages of Bahian cocoa plantations. Pedobiologia . v.39, p. 423-433, 1995. 
EITEN, G. Delimitação do conceito de Cerrado. Arquivos do jardim botânico, Rio de Janeiro, v.21, p.125-134, 1977.

EINTEN, G. Physiognomic categories of vegetation. In: MIYAWAKI A, BOGENRIEDER A, OKUDA $S$, WHITE J (eds). Vegetation ecology and creation of new environments. Tokio: Tokai Univ Pres,p. 387-403, 1987.

FERNÁNDEZ, F. Introducción a lãs Hormigas de la región Neotropical. Instituto de Investigación de Recursos Biológicos Alexander von Humboldt, Bogotá, Colombia. P.398, 2003.

FORTI, L.C \& BOARETTO, M, A, C. Formigas cortadeiras: Biologia, ecologia, e controle. São Paulo: UESB, p. 61, 1997.

FOWLER, H.G., FORTI, L.C. BRANDÃO, C.R.F.; DELABIE, J.H.C. \& VASCONCELOS, H.L. Ecologia nutricional de formigas. In: Panizzi, A.R. \& Parra, J.R.P. (Org). Ecologia nutricional de insetos e suas implicações no manejo de pragas. São Paulo: Manole Editora, p.131-223, 1991.

GOOGLE EARTH. Guia do usuário. Disponível em: Acesso em: 20/12/2013

HARIDASAN, M.. Aluminum accumulation by some Cerrado native species in Central Brazil. Plant and Soil 65: 265-273, 1982

KLINK \& MACHADO. A conservação do cerrado brasileiro. Megadiversidade, v1, Brasilia . p.148-145 . 2005.

KLINK, C. A. \& MOREIRA, A. D. Past and current human occupation, and land use. In: OLIVEIRA, P. S. \& MARQUIS, R. J. eds. The cerrados of Brazil: ecology and natural history of a Neotropical savanna. New York, Columbia University. p.69-90. 2002.

KRONKA F J N; NALON, M.A; MATSUKUMA C K KANASHIRO; MSHIZUE SHIN-IKE M M; LENI;Y. LIMA M P R; GUILLAUMON J R; BARRADAS; A M F; PAVÃO M; MANETTI L.A BORGO S C; Monitoramento da vegetação natural e do reflorestamento no Estado de São Paulo. Anais XII simpósio de brasileiro de sensoriamento remoto, Goiânia, Brasil, INPE, p.1569-157, 2005.

MACHADO, R. B. et al. Estimativas de perda da área do Cerrado brasileiro. Conservation International do Brasil, Brasília, 2004.

MARCHÃO, R.L. Soil macrofauna under integrated crolivestock systems in a Brazilian Cerrado Ferralsol. Pesquisa Agropecuária Brasileira, v.44, n.8, p.1011-1020, 2009. Disponível em: Acesso em: 21 ago. 2011.

MAJER, J .D. Ants:Bio-indicators of minesite rehabilitation land- use, and land conservation. Environmental Management, COLLEGE Park, v. 7, n. 4.,p. 375-383, 1983.

MARINHO C. GS; ZANETTI, R; DELABIE, J. H. C; MARCELO N. SCHLINDWEIN, M.N; RAMOS, L.S. Diversidades de Formigas (Hymenoptera: Formicidae) da Serapilheira em Eucaliptais (Myrtaceae) e Área de Cerrado de Minas Gerais. Neotropical Entomology 31(2). 2002. 
PARR, C.L. \& CHOWN, S.L. Inventory and bioindicator sampling: testing pitfall and Winkler methods with ants in a South African savanna. Journal of Insect Conservation, v. 5, p. 27-36, 2001.

PRIMACK, R.B.; RODRIGUES, E. Biologia da conservação. Londrina: Editora Planta, p.328, 2002.

REDFORD, K.H.There is more biodiversity than the tropical rain forests. conservation Biology. p.328-330, 1990.

RIBEIRO, J.F. \& B.M.T. WALTER. Fitofisionomias do bioma do cerrado, p. 89-152. In S.M. Sano \& S.P. Almeida (eds.), Cerrado: ambiente e flora. EMBRAPACPAC, 556p. 1998.

VEIGA, J.B.;SANTOS, R C, LOPES, M P M, SILVA, R R,; SILVA, A.C S \& OLIVEIRA A S. Avaliação rápida da riqueza de formigas (Hymenoptera, Formicidae) em fragmentos de floresta ombrófila na região de Alta Floresta, MT Revista de Ciências Agroambientais Alta Floresta, MT, UNEMAT, v.13, n.2, p.13-18, 2015

WILSON , E.O. The insects societies, , Behavioral ecology and sociobiology University de Harvard, Cambridge, Massachusetts, USA, 549p. 1971.

WILSON, E. O. The organization of colony defense in the Ant Pehidole dentate Mayr (Hymenoptera: Formicidae), Behavioral ecology and sociobiology, University of Harvard, Cambridge , Massachusetts, USA , p 63-81,1976. 